\title{
Towards an improved mass composition analysis with LOFAR
}

\author{
A. Corstanje ${ }^{* 1,2}$, S. Buitink ${ }^{1}$, A. Bonardi ${ }^{2}$, H. Falcke ${ }^{2,3,4}$, B.M. Hare ${ }^{5}$, \\ J. R. Hörandel ${ }^{2,3,1}$, T. Huege ${ }^{7,1}$, G. Krampah ${ }^{1}$, P. Mitra ${ }^{1}$, A. Nelles ${ }^{8,9}$, H. Pandya ${ }^{1}$, \\ J. P. Rachen ${ }^{1,2}$, L. Rossetto ${ }^{2}$, O. Scholten ${ }^{5,6}$, S. ter Veen ${ }^{2,4}$, T. N. G. Trinh ${ }^{5,10}$, \\ T. Winchen ${ }^{1}$
}

1 Astrophysical Institute, Vrije Universiteit Brussel, Pleinlaan 2, 1050 Brussels, Belgium

2 Department of Astrophysics/IMAPP, Radboud University Nijmegen, P.O. Box 9010, 6500 GL

Nijmegen, The Netherlands

3 NIKHEF, Science Park Amsterdam, 1098 XG Amsterdam, The Netherlands

4 Netherlands Institute of Radio Astronomy (ASTRON), Postbus 2, 7990 AA Dwingeloo, The Netherlands

5 KVI-CART, University Groningen, P.O. Box 72, 9700 AB Groningen, The Netherlands

6 Interuniversity Institute for High-Energy, Vrije Universiteit Brussel, Pleinlaan 2, 1050

Brussels, Belgium

7 Institut für Kernphysik, Karlsruhe Institute of Technology(KIT), P.O. Box 3640, 76021,

Karlsruhe, Germany

8 DESY, Platanenallee 6, 15738 Zeuthen, Germany

9 ECAP, Friedrich-Alexander-University Erlangen-Nürnberg, 91058 Erlangen, Germany 10 Department of Physics, School of Education, Can Tho University, Campus II, 3/2 Street, Ninh Kieu District, Can Tho City, Vietnam E-mail: a.corstanje@astro.ru.n 1

\begin{abstract}
The LOFAR radio telescope measures air showers in the energy range $10^{17}$ to $10^{18} \mathrm{eV}$. For each measured shower, the depth of shower maximum $X_{\max }$ is reconstructed by simulating the radio signal for an ensemble of showers using Corsika and CoREAS. Fitting their radio 'footprints' on the ground to the measured radio data yields an $X_{\max }$ estimate to a precision of about $20 \mathrm{~g} / \mathrm{cm}^{2}$. Compared to previous works, we have improved the method in several ways. Local atmospheric data and refractive index profiles are now included into the simulations. The energy estimate and the fitting procedure are now done using the radio signals only, thus limiting systematic uncertainties due to the particle detector array (LORA). Using selection criteria from a more elaborate characterisation of the radio and particle detection, we reduce a composition bias in the $X_{\max }$ reconstruction. A possible residual bias has been bounded from above. Thus, the systematic uncertainties on $\left\langle X_{\max }\right\rangle$ have been lowered, reducing an important limiting factor for composition studies at any level of statistics.
\end{abstract}

36th International Cosmic Ray Conference -ICRC2019-

July 24th - August 1st, 2019

Madison, WI, U.S.A.

${ }^{*}$ Speaker. 


\section{Introduction}

LOFAR is the most densely instrumented radio-based cosmic-ray observatory in the world. In the center of the array, air showers are detected by hundreds of antennas simultaneously, probing the intensity, shape and polarization of the radio footprint at the ground. These high-resolution measurements have played an important role in the understanding and verification of the radio emission mechanism of air showers. It also allows for reconstruction of the depth of shower maximum, $X_{\max }$, with a resolution of the order of $20 \mathrm{~g} / \mathrm{cm}^{2}$, comparable to the fluorescence detection technique. We have previously demonstrated that LOFAR is thus capable of studying mass composition in the energy range of $\sim 10^{17}-10^{18} \mathrm{eV}$. Since then, various improvements have been made to the antenna calibration, the accuracy of simulations and the reconstruction techniques. Here, we highlight the most important changes and discuss the achieved reduction in systematic uncertainties.

\section{Method}

We reconstruct $X_{\max }$ of each measured air shower that was detected by at least 3 LOFAR stations. The reconstruction is based on the procedure in [1]. To this end, we first simulate 600 showers with CONEX [2], which is very fast compared to a full CoREAS simulation. From this set, a subset of about 30 showers are selected which span the natural range of $X_{\max }$. Ten of these are chosen in an interval $\pm 20 \mathrm{~g} / \mathrm{cm}^{2}$ from a first estimate of $X_{\max }$ based on a parametrization of the radio footprint [3], to have a denser sampling there. These showers are fully simulated with Corsika [4] and CoREAS [5]

Their radio signal intensities are fitted to the measured LOFAR data, having the shower core position and an overall scale factor as free parameters. This gives a $\chi^{2}$-value as fit quality for each simulated shower. As shown for an example event in Fig. 1, an optimum is found using a parabolic fit in a range around the minimum $\chi^{2}$, and this is taken as the reconstructed $X_{\max }$.

The fit is now done on radio data only, in contrast to [1] where the particle detector signals were included as well. This performs about equally well, while removing systematic uncertainties related to particle-based event reconstruction. A small fraction of showers (on the order of 10\%) could not be accurately reconstructed without the particle detector signals, and these are now automatically discarded.

The primary energy of the shower is estimated from the measured radio intensity compared to the radio intensity of the best-fitting simulated shower. The radio intensity depends quadratically on the primary energy $[6,3]$. Therefore, the square root of the overall scale factor in the fit is used as a correction factor to the simulated energy. This method is now preferred, following a recent improvement in calibration the absolute scale of the measured radio signal [7]. Using the emission from the Galaxy as a calibration source, and implementing a model of the signal chain with its electronic noise contributions, allowed for a systematic uncertainty of $13 \%$ in amplitude. Statistical uncertainties are close to $10 \%$ on average.

Uncertainties on core position, energy and $X_{\max }$ are calculated from a Monte Carlo procedure on the simulated showers. We add to the simulated signals the noise levels measured in each antenna. For 3 realisations of the random noise, we produce a dataset which is reconstructed as if 

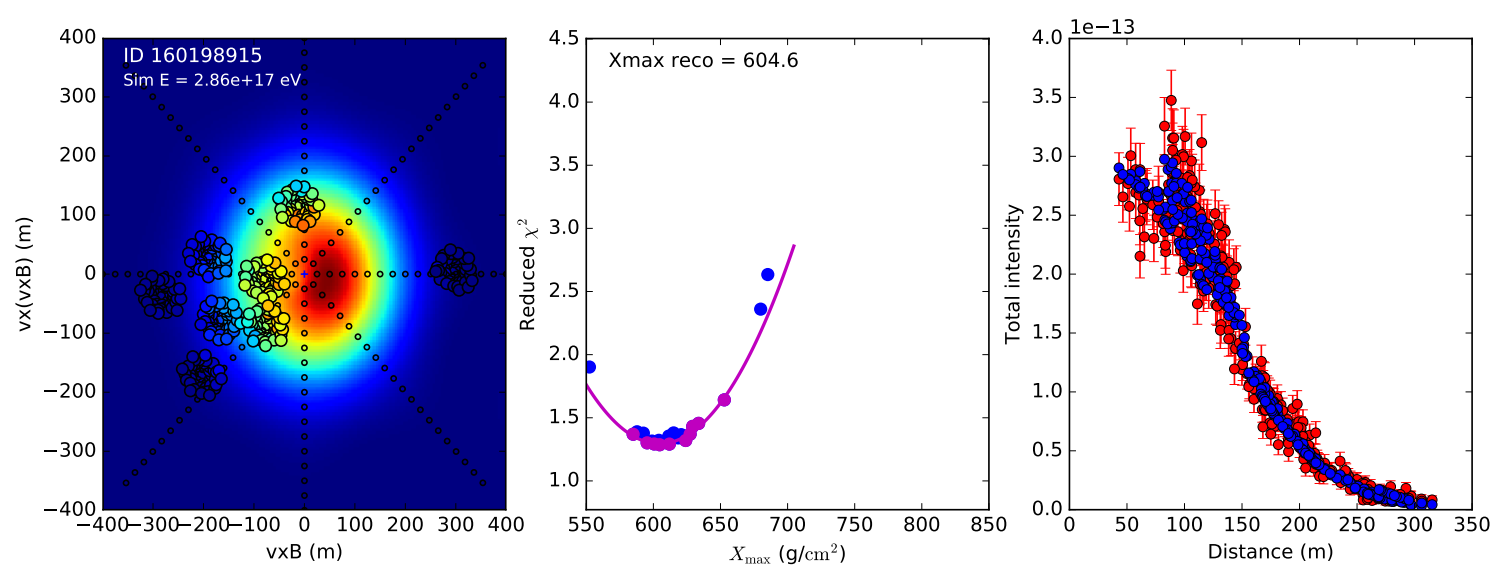

Figure 1: Left panel: signal intensity for best-fitting shower, with LOFAR measurements, in the shower plane. Middle panel: reduced $\chi^{2}$ as function of $X_{\max }$, with parabolic fit through the lower envelope of points. Right panel: projected 1-D lateral distribution, with the measurements as red points with margins, and the simulation values as blue points.

it were a measured shower (using the same code), using the other simulated showers as reconstruction ensemble. We compare the real core position, $X_{\max }$ and energy, which are known for the simulations, with the reconstructions thus found. Their RMS errors are taken as their uncertainties, which apply to the measured shower as well as to the ensemble of simulated showers.

\section{Sample selection criteria}

For a composition analysis based on $X_{\max }$ and energy of a set of measured air showers, it is critical that the dataset represents the $X_{\max }$-distribution in nature. Including all reconstructable measurements in the dataset typically leads to a biased sample. Due to the irregular layout of LOFAR, it is not straightforward to establish a fiducial volume in parameter space leading to direct inclusion/exclusion criteria for measured air showers, based on shower core, arrival direction, and energy. Especially for moderate-sized datasets of hundreds to thousands of showers, an analysis per shower is preferred.

The main sources of bias in $X_{\max }$ arise from the thresholds of the particle detector trigger and the LOFAR radio detection. The particle detectors will trigger more easily for showers penetrating deeper into the atmosphere, i.e. at high $X_{\max }$ values. On the other hand, the LOFAR radio detection threshold, which is set at 3 LOFAR stations having a significant signal in at least half of the antennas, is easier to reach for low- $X_{\max }$ showers. As these have a larger radio footprint, they have a higher likelihood of a detection in 3 LOFAR stations.

To ensure a bias-free sample, a sufficient main criterion is that each measured shower must be able to trigger both LOFAR and the particle detector array LORA, would it have had any other $X_{\max }$ in the natural range. Using the Monte Carlo (simulated) ensemble for each measured shower, this is tested, as described below. 


\subsection{Cuts on reconstruction quality}

From the Monte Carlo procedure outlined in Sect. 2, we obtain uncertainties on $X_{\max }$, energy and on the core position. These uncertainties apply to the simulated ensemble as a whole, as well as to the measured shower itself. By design, uncertainties calculated in this way are independent of $X_{\max }$, and therefore no composition bias will be introduced by placing quality cuts on these uncertainties. The uncertainty on the core position, being a basic geometric property of the shower, is used as an overall reconstruction quality indicator. Clearly, the uncertainties on $X_{\max }$ and core position are correlated; a cut around $7.5 \mathrm{~m}$ rejects most of the poorly reconstructed showers, and retains most of the good showers.

\subsection{Trigger test}

The simulated ensemble of about 30 showers provides a set of particles reaching the ground, from the Corsika simulation. We use a Geant4 [8] simulation of the particle detectors to check if these particle densities are sufficient to trigger LORA. Each detector will trigger if one or more particles pass through it; a coincidence of 13 out of 20 detectors is needed for a trigger. As this is subject to Poisson statistics, we require a trigger probability of at least $99 \%$.

Similarly, we require each shower in the simulated ensemble to be able to trigger 3 LOFAR stations. The core position from the best-fit reconstruction is assigned to all simulated showers. The measured noise level from the LOFAR data is taken as reference, and a signal-to-noise ratio of 6 (in intensity) is set as a condition to detect the signal for each antenna. From the data analysis pipeline, a station has a valid detection if at least half the antennas have a signal above this SNR.

\section{Results: test for residual bias}

We have tested for bias in our data sample, after the fiducial cuts described in Sect. 3, by evaluating the zenith dependence of the distribution of $X_{\max }$. A non-uniform value for $<X_{\max }>$ over zenith angle would point at unresolved systematic effects in the events reconstruction or detection probabilities. However, we first need to correct for a small dependence of average $\lg E$ on zenith angle, as shown in Fig. 2, left panel. To correct for this effect we introduce a parameter $Y$ for each shower:

$$
Y=X_{\max }+55(\lg (E)-17.4) \mathrm{g} / \mathrm{cm}^{2},
$$

the value of 17.4 being close to the average log-energy in our sample.

The results for $Y$ as a function of zenith angle are shown in the right panel of Fig. 2, together with a constant and a linear fit. The slope of the linear fit is $0.14 \pm 0.44$, which is compatible with zero. The uncertainty of the constant fit is $3.2 \mathrm{~g} / \mathrm{cm}^{2}$, which is added to the systematic uncertainties, as a bias at this level cannot be ruled out.

A complete scatter plot of $X_{\max }$ versus zenith angle is shown in Fig. 3, for a set of $N=298$ showers passing the reconstruction quality criterion. Of these, 196 also pass both fiducial selection criteria. 

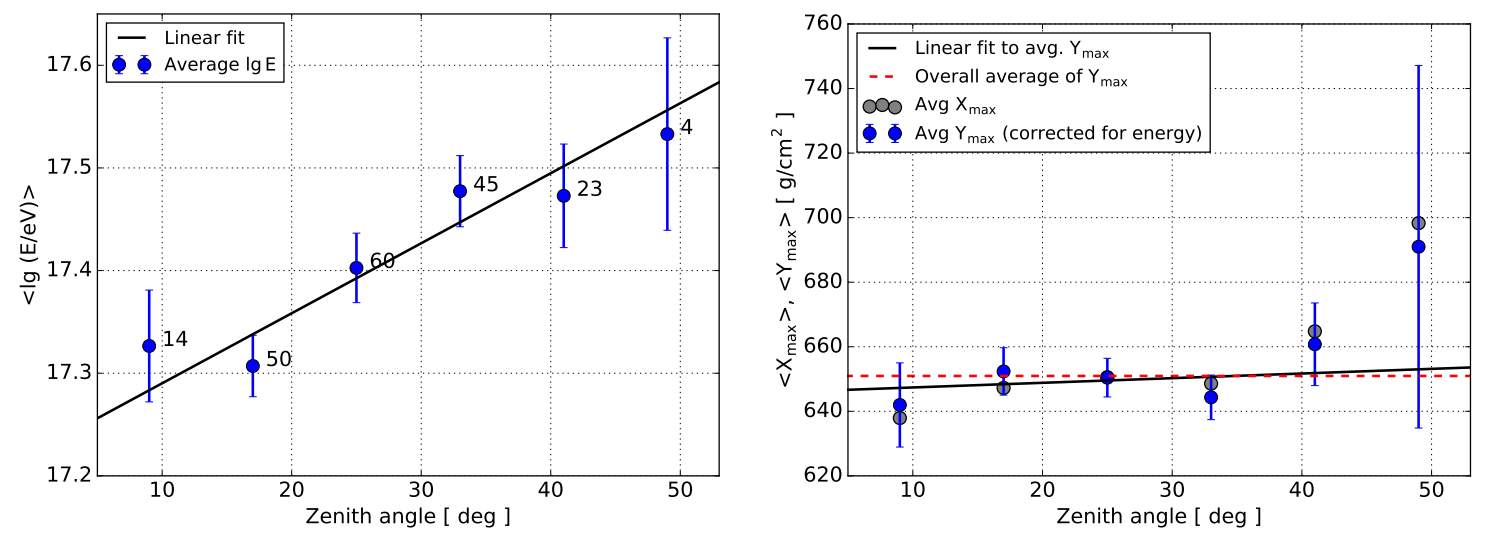

Figure 2: Left panel: The average $\log E$ as a function of zenith angle, with linear fit. Right panel: Average energy-corrected $X_{\max }$, defined as $Y$ in Eq. 4.1, versus zenith angle, with a constant and linear fit.

\section{Systematic uncertainties}

Systematic uncertainties in the reconstruction of $X_{\max }$ arise due to a number of factors. These are summarized in Table 1. Choosing a hadronic interaction model for the Corsika simulations gives a systematic uncertainty of about $5 \mathrm{~g} / \mathrm{cm}^{2}$ [9]; we have used QGSJetII-04 [10]. In the composition analysis, the average $X_{\max }$ for a given primary element and energy varies up to about $15 \mathrm{~g} / \mathrm{cm}^{2}$ compared to e.g. EPOS-LHC [11] and Sibyll-2.1 [12]. The latter is accounted for by doing the composition analysis separately using each model for the $X_{\max }$ distributions.

We have included local atmospheric profiles at the time of each measurement into the simulations [13]. This has reduced the systematic effects of density and refractive-index profiles from 4 to $11 \mathrm{~g} / \mathrm{cm}^{2}$ [14] to only $2 \mathrm{~g} / \mathrm{cm}^{2}$.

We have tested for a possible residual bias in our $X_{\max }$ sample (see Sect. 4); the amount found there has been added to the systematic uncertainties.

In the parabolic curve fit to obtain the optimum $X_{\max }$, we have reduced a possible systematic effect by ensuring that several showers have been simulated in a close region around the reconstructed $X_{\max }$.

In total, a systematic uncertainty on $X_{\max }$ of $7 \mathrm{~g} / \mathrm{cm}^{2}$ is found. This is comparable to the value of 7 to $11 \mathrm{~g} / \mathrm{cm}^{2}$ for the Pierre Auger Observatory in this energy range [15]. For the energy mea-

Table 1: Systematic uncertainies in the $X_{\max }$ reconstruction

\begin{tabular}{lll}
\hline \hline & Systematic uncertainty & Additional statistical uncertainty \\
\hline Choice of hadronic interaction model & $5 \mathrm{~g} / \mathrm{cm}^{2}$ & \\
Remaining atmospheric uncertainty & $\sim 1 \mathrm{~g} / \mathrm{cm}^{2}$ & $\sim 2 \mathrm{~g} / \mathrm{cm}^{2}$ \\
Five-layer atmosphere CORSIKA & $2 \mathrm{~g} / \mathrm{cm}^{2}$ & $4 \mathrm{~g} / \mathrm{cm}^{2}$ \\
Possible residual bias & $3.2 \mathrm{~g} / \mathrm{cm}^{2}$ & \\
Curve fit for $\chi^{2}$ optimum & $\leq 1 \mathrm{~g} / \mathrm{cm}^{2}$ & \\
\hline Total, added in quadrature & $7 \mathrm{~g} / \mathrm{cm}^{2}$ & \\
\hline \hline
\end{tabular}




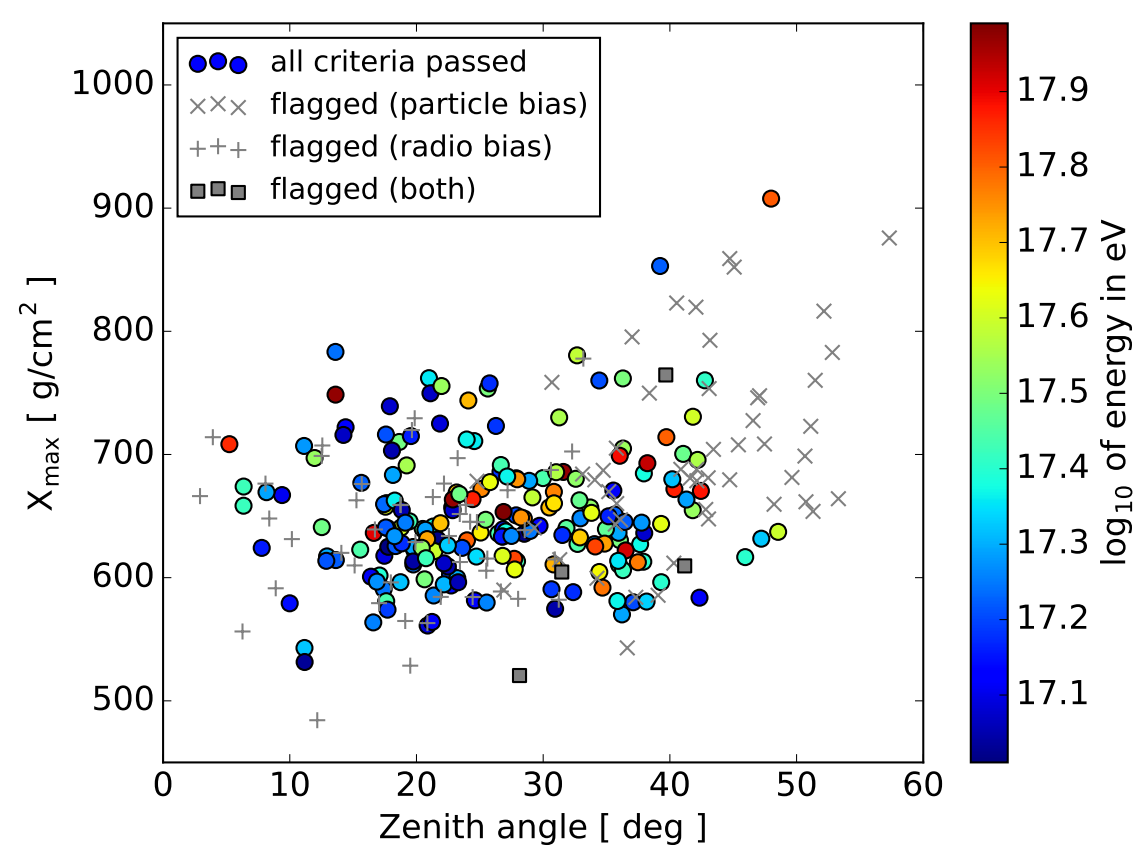

Figure 3: $X_{\max }$ versus zenith angle for 298 showers passing the reconstruction quality criterion. For those passing all criteria, the colored circles represent their energy. Showers rejected by the selection criteria for the particle trigger and the radio detection are shown as crosses and + symbols.

surement, the systematic uncertainty amounts to $14 \%$ [16], which is dominated by the uncertainty on the absolute scale of the radio calibration [7], with two smaller contributions added in quadrature: a contribution due to the choice of hadronic interaction model, and the invisible energy in air showers, of $4 \%$ [17], and a general contribution from using radiation energy from microscopic air shower simulations, of $2.6 \%[18,19]$. This is an improvement of about a factor 2 over using the LORA particle detector array for the energy, which has a $27 \%$ systematic uncertainty [20, 21].

\section{Summary}

We have improved the reconstruction method, by using radio data only for both the $X_{\max }$ and energy estimate. The latter is now available thanks to a better calibration of the LOFAR antennas. Local atmospheric profiles are included in the simulations for each shower. This leads to a systematic uncertainty in $X_{\max }$ of $7 \mathrm{~g} / \mathrm{cm}^{2}$, and $14 \%$ in energy. The statistical uncertainty in the energy is reduced from about 30 to $10 \%$. The fiducial selection criteria have been improved, mainly in the evaluation of the particle trigger threshold. We have tested for residual bias after the fiducial selection, by evaluating the zenith dependence of $X_{\max }$ in our dataset. No significant residual bias was found, and at our level of statistics, it is bounded by 3 to $4 \mathrm{~g} / \mathrm{cm}^{2}$. Thus, the current level of accuracy is sufficient for a refined composition analysis. 


\section{Acknowledgements}

The LOFAR cosmic-ray key science project acknowledges funding from an Advanced Grant of the European Research Council (FP/2007-2013) / ERC Grant Agreement n. 227610. The project has also received funding from the European Research Council (ERC) under the European Union's Horizon 2020 research and innovation programme (grant agreement No 640130). We furthermore acknowledge financial support from FOM, (FOM-project 12PR304). TW is supported by DFG grant WI 4946/1-1. AN is supported by the DFG grant NE 2031/2-1. LOFAR, the Low Frequency Array designed and constructed by ASTRON, has facilities in several countries, that are owned by various parties (each with their own funding sources), and that are collectively operated by the International LOFAR Telescope foundation under a joint scientific policy.

\section{References}

[1] S. Buitink et al., Method for high precision reconstruction of air shower $X_{\max }$ using two-dimensional radio intensity profiles, Phys. Rev. D 90 (Oct, 2014) 082003.

[2] T. Bergmann et al., One-dimensional hybrid approach to extensive air shower simulation, Astroparticle Physics 26 (2007), no. 6420 - 432.

[3] A. Nelles et al., A parameterization for the radio emission of air showers as predicted by CoREAS simulations and applied to LOFAR measurements, Astroparticle Physics 60 (2015) 13 - 24.

[4] D. Heck et al., CORSIKA: a Monte Carlo code to simulate extensive air showers. Feb., 1998.

[5] T. Huege, M. Ludwig, and C. W. James, Simulating radio emission from air showers with CoREAS, AIP Conference Proceedings 1535 (2013), no. 1 128-132.

[6] B. Zimmermann et al., Cosmic ray physics with the Auger Engineering Radio Array (AERA), Nuclear and Particle Physics Proceedings 291-293 (2017) 90 - 95. New eyes on the Universe, CRIS 2016 Cosmic Rays International Seminars Proceedings of the Cosmic Rays International Seminars.

[7] K. Mulrey et al., Calibration of the LOFAR low-band antennas using the Galaxy and a model of the signal chain, accepted by Astroparticle Physics (2019) (2019). Arxiv:1903.05988.

[8] S. Agostinelli et al., Geant4: a simulation toolkit, Nuclear Instruments and Methods in Physics Research Section A: Accelerators, Spectrometers, Detectors and Associated Equipment 506 (2003), no. $3250-303$.

[9] S. Buitink et al., A large light-mass component of cosmic rays at $10^{17}-10^{17.5}$ electronvolts from radio observations, Nature (Mar, 2016) 70 .

[10] S. Ostapchenko, QGSJET-II: physics, recent improvements, and results for air showers, EPJ Web of Conferences 52 (2013) 02001

[11] T. Pierog, I. Karpenko, J. M. Katzy, E. Yatsenko, and K. Werner, EPOS LHC: test of collective hadronization with data measured at the CERN Large Hadron Collider, Phys. Rev. C 92 (Sep, 2015) 034906.

[12] E.-J. Ahn, R. Engel, T. K. Gaisser, P. Lipari, and T. Stanev, Cosmic ray interaction event generator SIBYLL 2.1, Phys. Rev. D 80 (Nov, 2009) 094003.

[13] P. Mitra et al., Reconstructing air shower parameters with LOFAR using event specific GDAS atmospheres, submitted to Astroparticle Physics (2019). 
[14] A. Corstanje et al., The effect of the atmospheric refractive index on the radio signal of extensive air showers, Astroparticle Physics 89 (2017) 23 - 29.

[15] J. Bellido et al., Depth of maximum of air-shower profiles at the Pierre Auger Observatory: Measurements above $10^{17.2} \mathrm{eV}$ and Composition Implications, Proceedings of the 35th International Cosmic Ray Conference, Busan, Korea, PoS (2017) 506.

[16] K. Mulrey et al., The energy scale of cosmic rays detected with LOFAR, These proceedings (2019).

[17] A. Aab et al., Data-driven estimation of the invisible energy of cosmic ray showers with the Pierre Auger Observatory, submitted to Phys. Rev. D; arXiv:1901.08040 (2019).

[18] C. Glaser, M. Erdmann, J. Hörandel, T. Huege, and J. Schulz, Simulation of the radiation energy release in air showers, EPJ Web Conf. 135 (2017) 01016.

[19] M. Gottowik, C. Glaser, T. Huege, and J. Rautenberg, Determination of the absolute energy scale of extensive air showers via radio emission: systematic uncertainty of underlying first-principle calculations, Astroparticle Physics 103 (2018) 87 - 93.

[20] S. Thoudam et al., LORA: A scintillator array for LOFAR to measure extensive air showers, Nuclear Instruments and Methods in Physics Research Section A: Accelerators, Spectrometers, Detectors and Associated Equipment 767 (2014) 339 - 346.

[21] S. Thoudam et al., Measurement of the cosmic-ray energy spectrum above $10^{16} \mathrm{eV}$ with the LOFAR Radboud Air Shower Array, Astroparticle Physics 73 (2016) 34 - 43. 